\title{
Leveraging Integrated Primary Care to Address Patients' and Families' Unmet Social Needs: Aligning Practice with National Academy of Sciences, Engineering and Medicine Recommendations
}

\author{
Ellen Poleshuck, PhD, Kyle Possemato, PhD, Emily M. Johnson, PhD, \\ Alicia J. Cohen, MD, MSc, FAAFP, Colleen T. Fogarty, MD, MSc, FAAFP, and \\ Jennifer S. Funderburk, $P b D$
}

Primary care is well-poised to address unmet social needs that affect health. Integrated primary care is increasingly common and can be leveraged to facilitate identification of practice and clinician-level modifiable characteristics and assist practices to address unmet social needs for patients and families. A recent National Academies of Sciences, Engineering, and Medicine (NASEM)'s consensus report identified 5 critical system-level activities to facilitate the integration of addressing social needs into health care: awareness (ask patients), adjustment (flexible intervention delivery), assistance (intervention to address the social need), alignment (link with community resources), and advocacy (policy change). This article outlines how integrated primary care characteristics, such as routine screening, functional workflows, interprofessional team communication, and patient-centered practices, exemplify the NASEM report's activities and offer robust biopsychosocial tools for addressing social needs. We provide a case to illustrate how these strategies might be used in practice. ( $\mathrm{J}$ Am Board Fam Med 2022;35:185-189.)

Keywords: Integrated Delivery of Health Care, Patient Care Team, Patient-Centered Care, Primary Health Care, Social Determinants of Health, Social Needs, Social Risk Factors, Team-Based Care

Social determinants of health are critical to health, and unmet social needs interfere with achievement of optimal health. ${ }^{1}$ Within the health care system, and, in particular, within primary care, our commitment to addressing social needs is evident while our understanding of how to respond to unmet social

This article was externally peer reviewed.

Submitted 12 July 2021; revised 22 September 2021; accepted 24 September 2021.

From the Department of Veterans Affairs, VA Center for Integrated Healthcare, Syracuse VA Medical Center, Syracuse, NY (EP, KP, EMJ, JSF); Departments of Psychiatry and Obstetrics \& Gynecology, University of Rochester Medical Center, Rochester, NY (EP); Department of Psychology, Syracuse University, Syracuse NY (KP); Veterans Affairs Center of Innovation in Long Term Services and Supports, VA Providence Healthcare System, Providence, RI (AJC); Department of Family Medicine, Alpert Medical School of Brown University, Providence, RI (AJC); Department of Health Services, Policy, and Practice, Brown University School of Public Health, Providence, RI (AJC); Department of Family Medicine, University of Rochester Medical Center, needs continues to evolve. ${ }^{2-9}$ Efforts to provide biopsychosocial primary care include screening for and implementing interventions to address homelessness, food insecurity, and lack of transportation. Centers for Medicare and Medicaid Service's Accountable Health Communities Model initiative, for example, aims to enhance clinical-community linkages through screening, referral, and community navigation services and is evaluating if this will

Rochester, NY (CTF, JSF); Department of Psychiatry, University of Rochester Medical Center, Rochester, NY (JSF).

Funding: This material is based upon work supported by the Department of Veterans Affairs, Veterans Health Administration, Office of Research and Development and Center for Integrated Healthcare.

Conflict of interest: The authors have no conflicts of interest to declare.

Corresponding author: Ellen Poleshuck, 300 Crittenden Blvd, University of Rochester Medical Center, Rochester, NY 14642

(E-mail: Ellen_Poleshuck@urmc.rochester.edu). 
improve health outcomes and reduce costs. ${ }^{10}$ There are many challenges to addressing social needs, however: effectiveness of social interventions for health, utilization, and cost outcomes to date are mixed, ${ }^{11}$ systemic racism pervades primary care and exacerbates social inequities and mistrust of the health care system, ${ }^{12}$ and primary care patient interest in $^{13}$ and uptake of ${ }^{14}$ social care assistance is limited. The complexity surrounding how to address unmet social needs in primary care also is due to the many interventions at policy, community, health care system, health care teams, individual clinician, family, and individual patient levels. ${ }^{15}$

We propose integrated primary care can be expanded to incorporate social needs. Primary care team and clinician-level characteristics can be leveraged to improve outcomes. An integrated primary care approach builds on health systems' efforts to optimize structures and processes to improve patients' experiences of health care by providing close collaboration and communication using a team of clinicians with diverse skills (eg, physicians, nurses, psychotherapists, social workers). ${ }^{16,17}$ This goal is accomplished with a comprehensive treatment plan to address patients' identified biopsychosocial needs based on their needs and preferences. This commentary describes how primary care can improve access and person-centered patient care for unmet social needs by targeting modifiable practice-level characteristics fundamental to integrated care. ${ }^{18}$

The National Academies of Sciences, Engineering, and Medicine (NASEM)'s expert committee recommended 5 system-level activities as critical to addressing social needs in health care: awareness (ask patients), adjustment (flexible intervention delivery), assistance (intervention to address the social need), alignment (connect with community resources), and advocacy (policy change). ${ }^{3}$ Integrated health care team members improve personcentered care and share responsibility directly for 4 of the 5 NASEM activities as highlighted in the following examples by improving person-centered access to care:

a. Awareness: eliciting patient social needs, priorities, and values as part of screening and assessment promotes awareness of patient context and preferences

b. Adjustment: considering barriers to engagement and offering flexibility, accommodation, and adjustment in how the interventions are delivered (eg, which team member; in-person/telehealth; frequency of visits) builds trust and safety

c. Assistance: warm handoffs and co-location of social services (eg, food boxes, transportation vouchers) facilitates patients accessing desired assistance

d. Alignment: partnering with community-based agencies to establish alignment to provide coordinated care to the patient and emphasizing continuity and follow-up after the plan is initiated (Table 1).

Using a biopsychosocial approach, an integrated team can address physical health, mental health, and social needs based on the patient's priorities and goals and achieve a plan acceptable to the patient and family. While our focus here on horizontal integration within practices emphasizes how primary care can provide more coordinated and collaborative planning, consideration of vertical pathways to facilitate health system integration activities for specific diseases is also critical.

Team-based care facilitates primary care practices' capacity to incorporate a more robust, patient-centered comprehensive treatment plan which addresses patients' social needs. Achieving a shared agenda and plan which addresses social needs requires the integrated primary care team to work together in a coordinated, flexible way toward shared goals resulting in value-added outcomes. ${ }^{19}$ This high-level of effectiveness can be accomplished via: (1) relationships between interprofessional team members that include formal and informal conversations (eg, team huddles, impromptu collaborations); (2) clearly delineated workflows that focus on a shared goal (ie, unmet social needs); (3) willingness and ability to identify patients' priorities, especially as they relate to social needs, and modify the shared plan; (4) explicit discussion and agreement on the responsibilities of the patient, family, and health professionals in the patient's health care plan; (5) clear communication between team members and with patients; and, perhaps most importantly, (6) safe, trusting, respectful relationships in which the patient feels understood, supported, and respected by the health care team. Early research shows integrated health care strategies like warm handoffs and continuity in the patient/clinician relationship, for example, are associated with improved uptake of tobacco cessation interventions and cancer screenings. ${ }^{20,21}$ Further, some patients find it sufficient if their clinician is 


\begin{tabular}{|c|c|}
\hline System Level Activity & Integrated Primary Care Strategy \\
\hline $\begin{array}{l}\text { Awareness: Activities that identify the social risks and assets of } \\
\text { defined patients and populations. }\end{array}$ & $\begin{array}{l}\text { - Routine screening of social needs } \\
\text { - Formal and informal team communication (eg, electronic } \\
\text { health record templates, flow sheets and best practice alerts; } \\
\text { team huddles; warm handoffs and informal conversations) } \\
\text { - Assessment of patient priorities }\end{array}$ \\
\hline $\begin{array}{l}\text { Adjustment: Activities that focus on altering clinical care to } \\
\text { accommodate identified social barriers. }\end{array}$ & $\begin{array}{l}\text { - Evaluate structural and patient-level barriers } \\
\text { - Shared decision making to develop action plan } \\
\text { - Respect patient preference for which team member the } \\
\text { patient meets with and how often } \\
\text { - Options for intervention modality (in-person; text; } \\
\text { telehealth) }\end{array}$ \\
\hline $\begin{array}{l}\text { Assistance: Activities that reduce social risk by providing assistance } \\
\text { in connecting patients with relevant social care resources. }\end{array}$ & $\begin{array}{l}\text { - Functional workflows for responding to identified needs } \\
\text { - Initiate response during the visit } \\
\text { - On-site provision of intervention to address social need } \\
\text { (preferable) or active connection of patient to community } \\
\text { resource }\end{array}$ \\
\hline $\begin{array}{l}\text { Alignment: Activities undertaken by health care systems to be } \\
\text { aware of and connect with existing social care assets in the } \\
\text { community, organize them to facilitate synergies, and invest in } \\
\text { and deploy them to positively affect health outcomes. }\end{array}$ & $\begin{array}{l}\text { - Development of shared agenda across the practice } \\
\text { - Staff training } \\
\text { - Clear delineation of roles and responsibilities } \\
\text { - Team meetings and huddles } \\
\text { - Patient education materials describing team member roles } \\
\text { - Relational continuity } \\
\text { - Follow-up to re-assess and recalibrate plan }\end{array}$ \\
\hline
\end{tabular}

simply cognizant of their life context and social situation. $^{22}$

While primary care clinicians may be suitably trained, desiring, and prepared to expand their role to address social needs, progress will be limited until viable business models can be established and health care systems agree with this stance. Thus, Advocacy, the fifth activity, is required to establish policies that incentivize strategies to support social needs. Critical examples include a population health approach to evaluating costs effectiveness, payment for screening and diagnosis of social needs, remuneration for activities like warm handoffs and social need interventions, and direct reimbursement for social worker and community health worker activities.

Consider this case example that, while simplistic for purposes of brevity, highlights the clinician and team characteristics regarding a patient with social needs: Sylvia White is 34 years old and has type 2 diabetes, chronic pelvic pain, and a long-standing history of depression and posttraumatic stress disorder. Her presenting concern is her chronic pain; she finds it increasingly difficult to continue working as a home health aide. Ms. White has a 12-year-old son and 10- and 8-year-old daughters, the youngest of whom has autism spectrum disorder.
She recently ended her relationship with the children's father due to intimate partner violence and is staying with her sister. Using the recommendations outlined, we offer suggestions on how to address social needs within her health care. First, the medical assistant, nurse, and primary care clinician note during the huddle that all team members (eg, embedded social or community health workers) are accepting warm handoffs today. Next, someone at the front desk explains to Ms. White that the practice is screening for social needs because they are viewed as an important part of health and hands her a tablet with standardized screening questions (see SIREN website for screening instrument options $^{23}$ ) The medical assistant who rooms the patient reviews the screening results and encourages the patient to discuss her needs during her appointment. Next, the primary care clinician acknowledges the needs she identified, validates her decision to stay with her sister, and asks how her current needs affect her chronic pain and diabetes management (awareness of unstable housing and employment; unsafe relationship). This serves to open the conversation and demonstrates empathy for Ms. White's context. Determining if Ms. White has an existing trusting relationship with the practice's social worker 
or community health worker and inviting her to meet with the person she trusts most sets the stage for a warm handoff, page, or phone call to create relational continuity (assistance with accessing resources). Engaging Ms. White in a discussion about her goals and priorities allows for shared decision-making regarding next steps (adjustment with prioritizing legal consultation and financial resources). Explicitly acknowledging factors that may impact her health care experience and her preferences for a plan, such as systemic racism and past negative experiences with social resource agencies, can invite her to share her own values and needs. A huddle, sharing Ms. White's plan across the clinical care team plus follow-up with her by the same person who partnered with her to develop the plan via calls, texts, or home visits, strengthens the trust and proactively anticipates potential barriers. Finally, having a member of Ms. White's care team reach out to the community agency providing intimate partner violence support to her (with her consent) and assessing changes in her social needs status and social resource engagement (alignment with community agency services) at her next appointment, along with her physical and mental health status, emphasizes the communication, teambased approach, and importance of discussing social needs as part of the appointment. The fifth NASEM activity, advocacy, could entail Ms. White's clinicians writing letters to support policies to improve reimbursement models for the coordinated, complex care provided.

Primary care practices need systemic, practice, and clinician-level strategies to respond to unmet social needs. Integrated primary care can provide the necessary expertise and skills. This article's recommendations can be used to maximize primary care team responses. Further research is needed to establish evidence for the most important team and clinician-level targets to facilitate interventions that will address patients' social needs within integrated primary care as part of whole-person care. Ultimately, place patients and their priorities at the center of their care. In this way, patients will find their biopsychosocial concerns supported, and they will be better able to access the care they need and want, improving their overall health.

The views expressed in this article are those of the authors. They do not necessarily reflect the position or policy of the
Department of Veterans Affairs or the United States government.

To see this article online, please go to: http://jabfm.org/content/ 35/1/185.full.

\section{References}

1. Marmot M. The health gap: doctors and the social determinants of health. Scand J Public Health 2017;45:686-93.

2. Committee on the Recommended Social and Behavioral Domains and Measures for Electronic Health Records, Board on Population Health and Public Health Practice, Institute of Medicine. Capturing Social and Behavioral Domains and Measures in Electronic Health Records. National Academies Press; 2014.

3. National Academies of Sciences, Engineering, and Medicine. Integrating Social Care into the Delivery of Health Care: Moving Upstream to Improve the Nation's Health. Washington, DC: The National Academies Press; 2019.

4. Council on Community Pediatrics, American Academy of Pediatrics. Poverty and child health in the United States. Pediatrics 2016;137:e20160339.

5. Alley DE, Asomugha CN, Conway PH, Sanghavi DM. Accountable health communities - addressing social needs through Medicare and Medicaid. N Engl J Med 2016;374:8-11.

6. US Department of Health and Human Services. Healthy People 2030: Social Determinants of Health. Updated August 2, 2021. Accessed November 29, 2021. Available at: https://health.gov/healthypeople/objectivesand-data/social-determinants-health.

7. ACOG Committee Opinion No. 729. American College of Obstetricians and Gynecologists. Importance of social determinants of health and cultural awareness in the delivery of reproductive health care. Obstet Gynecol 2018;131:e43-e48.

8. Etz RS, Cohen DJ, Woolf SH, et al. Bridging primary care practices and communities to promote healthy behaviors. Am J Prev Med 2008;35:S390-7.

9. Evans JM, Baker GR, Berta W, Barnsley J. The evolution of integrated health care strategies. Adv Health Care Manag 2013;15:125-61.

10. Centers of Medicare and Medicaid Services. Accountable Health Communities Model. Updated October 20, 2021. Accessed November 29, 2021. Available at: https://innovation.cms.gov/innovation-models/ahcm.

11. Gottlieb LM, Wing H, Adler NE. A systematic review of interventions on patients' social and economic needs. Am J Prev Med 2017;53:719-29.

12. Williams DR, Cooper LA. Reducing racial inequities in health: using what we already know to take action. IJERPH 2019;16:606.

13. Gruß I, Varga A, Brooks N, Gold R, Banegas MP. Patient interest in receiving assistance with self- 
reported social risks. J Am Board Fam Med 2021; 34:914-24.

14. De Marchis EH, Alderwick H, Gottlieb LM. Do patients want help addressing social risks? J Am Board Fam Med 2020;33:170-5.

15. Fichtenberg CM, Alley DE, Mistry KB. Improving social needs intervention research: Key questions for advancing the field. Am J Prev Med 2019;57: S47-S54.

16. Integrated Health Care. American Psychological Association. Published 2013. Accessed June 16, 2021. https://www.apa.org/health/integrated-healthcare.

17. Evans JM, Baker GR. Shared mental models of integrated care: aligning multiple stakeholder perspectives. J Health Organ Manag 2012;26: 713-36.

18. Sofaer S. Navigating poorly charted territory: patient dilemmas in health care "nonsystems". Med Care Res Rev 2009;66:75S-93S.
19. Salas E, Sims DE, Burke CS. Is there a "Big Five" in Teamwork? Small Group Research 2005;36:555-99.

20. Mussulman LM, Faseru B, Fitzgerald S, Nazir N, Patel V, Richter K. A randomized controlled pilot study of warm handoff versus fax referral for hospital-initiated smoking cessation among people living with HIV/AIDS. Addict Behav 2018;78:205-8.

21. O'Malley AS, Mandelblatt J, Gold K, Cagney KA, Kerner J. Continuity of care and the use of breast and cervical cancer screening services in a multiethnic community. Arch Intern Med 1997;157:1462-70.

22. Byhoff E, De Marchis EH, Hessler D, et al. Part II: a qualitative study of social risk screening acceptability in patients and caregivers. Am J Prev Med 2019;57:S38-S46.

23. Social Interventions Research \& Evaluation Network. Social Needs Screening Tool Comparison Table. 2019. Accessed November 29, 2021. Available at: https://sirenetwork.ucsf.edu/tools-resources/resources/ screening-tools-comparison. 